\title{
Combinatorial Formulas for Macdonald and Hall-Littlewood Polynomials
}

\author{
Cristian Lenart
}

Department of Mathematics and Statistics, State University of New York at Albany, Albany, NY 12222

\begin{abstract}
A breakthrough in the theory of (type $A$ ) Macdonald polynomials is due to Haglund, Haiman and Loehr, who exhibited a combinatorial formula for these polynomials in terms of fillings of Young diagrams. Recently, Ram and Yip gave a formula for the Macdonald polynomials of arbitrary type in terms of the corresponding affine Weyl group. In this paper, we show that a Haglund-Haiman-Loehr type formula follows naturally from the more general Ram-Yip formula, via compression. Then we extend this approach to the Hall-Littlewood polynomials of type $C$, which are specializations of the corresponding Macdonald polynomials at $q=0$. We note that no analog of the Haglund-Haiman-Loehr formula exists beyond type $A$, so our work is a first step towards finding such a formula.
\end{abstract}

Keywords: Macdonald polynomials, Hall-Littlewood polynomials, Haglund-Haiman-Loehr formula, alcove walks, Ram-Yip formula, Schwer's formula.

\section{Introduction}

Macdonald [14, 15] defined a remarkable family of symmetric orthogonal polynomials depending on parameters $q, t$, which bear his name. These polynomials generalize several other symmetric polynomials related to representation theory. For instance, at $q=0$, the Macdonald polynomials specialize to the Hall-Littlewood polynomials (or spherical functions on $p$-adic groups), and they further specialize to the Weyl characters (upon setting $t=0$ as well). There has been considerable interest recently in the combinatorics of Macdonald polynomials. This stems in part from a combinatorial formula for the ones corresponding to type $A$, which is due to Haglund, Haiman, and Loehr [4], and which is in terms of fillings of Young diagrams. This formula uses two statistics on the mentioned fillings, called "inv" and "maj". The Haglund-Haiman-Loehr formula already found important applications, such as new proofs of the Schur positivity for Macdonald polynomials [1, 3]. Let us also note that there is a version of the Haglund-Haiman-Loehr formula for the non-symmetric Macdonald polynomials [5], as well as a different formula for these polynomials due to Lascoux [7].

Schwer [18] gave a formula for the Hall-Littlewood polynomials of arbitrary type. Throughout this paper, we use the version of Schwer's formula that was derived by Ram [16]. Schwer's formula is in terms of so-called alcove walks, which originate in the work of Gaussent-Littelmann [2] and of the author with Postnikov [10, 11] on discrete counterparts to the Littelmann path model [12, 13]. Schwer's formula was recently generalized by Ram and Yip to a similar formula for the Macdonald polynomials [17]. The generalization consists in the fact that the latter formula is in terms of alcove walks with both "positive" and "negative" foldings, whereas in the former only "positive" foldings appear.

In this paper, we relate the Ram-Yip formula to the Haglund-Haiman-Loehr formula. More precisely, we show that we can group the terms in the type $A$ version of the Ram-Yip formula into equivalence classes, such that the sum in each class is a term in a new formula, which is similar to the Haglund-Haiman-Loehr one but contains considerably fewer terms, see [9]. An equivalence class consists of all the terms corresponding to alcove walks that produce the same filling of a Young diagram $\lambda$ (indexing the Macdonald polynomial) via a simple construction. In fact, in this paper we require that the partition $\lambda$ is a regular weight; the general case will be considered elsewhere. 
Our approach has the advantage of deriving the Haglund-Haiman-Loehr statistics "inv" and "maj" on fillings of Young diagrams in a natural way, from more general concepts. It also has the advantage of being applicable to other root systems, where no analog of the Haglund-Haiman-Loehr formula exists. As a first step in this direction, we derive here a formula in terms of fillings of Young diagrams for the Hall-Littlewood polynomials of type $C$ indexed by a regular weight; we proceed by compressing the type $C$ version of Schwer's formula. A completely similar formula exists in type $B$, while type $D$ is slightly more complex.

The structure of this extended abstract is as follows. In Section 2 we present our formula of Haglund-HaimanLoehr type for the Macdonald polynomials of type A. In Section 3 we present our new formula for the HallLittlewood polynomials of type $C$ in terms of fillings of Young diagrams. In Section 4 we give background information on root systems, alcove walks, the Ram-Yip formula, and Schwer's formula. In Section 5 we specialize the Ram-Yip formula to type $A$ and explain how it compresses to our formula for the corresponding Macdonald polynomials. In Section 6 we specialize Schwer's formula to type $C$ and explain how it compresses to our formula for the corresponding Hall-Littlewood polynomials. The full length versions of sections 5 and 6 are [9] and [8], respectively.

\section{A new formula of Haglund-Haiman-Loehr type}

In this section we present a new formula for the Macdonald polynomials of type $A$ that is similar to the HaglundHaiman-Loehr one [4]. This formula will be derived by compressing the Ram-Yip formula [17]. It also turns out that the new formula has considerably fewer terms even than the Haglund-Haiman-Loehr formula.

Let us consider a partition with $n-1$ distinct parts $\lambda=\left(\lambda_{1}>\lambda_{2}>\ldots>\lambda_{n-1}>0\right)$ for a fixed $n$ (this corresponds to a dominant regular weight for the root system of type $\left.A_{n-1}\right)$. Using standard notation, one defines $n(\lambda):=\sum_{i}(i-1) \lambda_{i}$. We identify $\lambda$ with its Young (or Ferrers) diagram, as usual, and denote by $(i, j)$ the cell in row $i$ and column $j$, where $1 \leq j \leq \lambda_{i}$. We draw this diagram in "Japanese style", that is, we embed it in the third quadrant, as shown below:

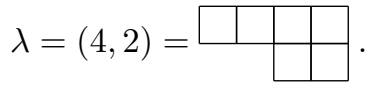

For any cell $u=(i, j)$ of $\lambda$ with $j \neq 1$, denote the cell $v=(i, j-1)$ directly to the right of $u$ by $\mathrm{r}(u)$.

Two cells $u, v \in \lambda$ are said to attack each other if either

(i) they are in the same column: $u=(i, j), v=(k, j)$; or

(ii) they are in consecutive columns, with the cell in the left column strictly above the one in the right column: $u=(i, j), v=(k, j-1)$, where $i<k$.

Remark 2.1 The main difference in our approach compared to the Haglund-Haiman-Loehr one is in the definition of attacking cells; note that in [4] these cells are defined similarly, except that $u=(i, j)$ and $v=(k, j-1)$ with $i>k$ attack each other.

A filling is a function $\sigma: \lambda \rightarrow[n]:=\{1, \ldots, n\}$ for some $n$, that is, an assignment of values in $[n]$ to the cells of $\lambda$. As usual, we define the content of a filling $\sigma$ as content $(\sigma):=\left(c_{1}, \ldots, c_{n}\right)$, where $c_{i}$ is the number of entries $i$ in the filling, i.e., $c_{i}:=\left|\sigma^{-1}(i)\right|$. The monomial $x^{\operatorname{content}(\sigma)}$ in the variables $x_{1}, \ldots, x_{n}$ is then given by $x^{\operatorname{content}(\sigma)}:=x_{1}^{c_{1}} \ldots, x_{n}^{c_{n}}$.

Definition 2.2 A filling $\sigma: \lambda \rightarrow[n]$ is called non-attacking if $\sigma(u) \neq \sigma(v)$ whenever $u$ and $v$ attack each other. Let $\mathcal{T}(\lambda, n)$ denote the set of non-attacking fillings.

Definition 2.3 Given a filling $\sigma$ of $\lambda$, let

$$
\begin{aligned}
& \operatorname{Des}(\sigma):=\{(i, j) \in \lambda:(i, j+1) \in \lambda, \quad \sigma(i, j)>\sigma(i, j+1)\}, \\
& \operatorname{Diff}(\sigma):=\{(i, j) \in \lambda:(i, j+1) \in \lambda, \quad \sigma(i, j) \neq \sigma(i, j+1)\} .
\end{aligned}
$$


We define a reading order on the cells of $\lambda$ as the total order given by considering the columns from right to left (largest to smallest), and by reading each column from top to bottom. Note that this is a different reading order than the usual (French or Japanese) ones.

Definition 2.4 An inversion of $\sigma$ is a pair $(u, v)$ of attacking cells, where u precedes $v$ in the considered reading order and $\sigma(u)>\sigma(v)$. Let $\operatorname{Inv}(\sigma)$ denote the set of inversions of $\sigma$.

Here are two examples of inversions, where $a<b$ :

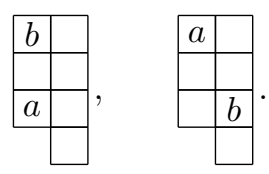

The arm of a cell $u \in \lambda$ is the number of cells strictly to the left of $u$ in the same row; similarly, the leg of $u$ is the number of cells strictly below $u$ in the same column.

Definition 2.5 The maj and inv statistics on fillings $\sigma$ are defined by

$$
\operatorname{maj}(\sigma):=\sum_{u \in \operatorname{Des}(\sigma)} \operatorname{arm}(u), \quad \operatorname{inv}(\sigma):=|\operatorname{Inv}(\sigma)|-\sum_{u \in \operatorname{Des}(\sigma)} \operatorname{leg}(u) .
$$

We are now ready to state a new combinatorial formula for the Macdonald $P$-polynomials in the variables $X=\left(x_{1}, \ldots, x_{n}\right)$.

Theorem 2.6 Given a partition $\lambda$ with $n-1$ distinct parts, we have

$$
P_{\lambda}(X ; q, t)=\sum_{\sigma \in \mathcal{T}(\lambda, n)} t^{n(\lambda)-\operatorname{inv}(\sigma)} q^{\operatorname{maj}(\sigma)}\left(\prod_{u \in \operatorname{Diff}(\sigma)} \frac{1-t}{1-q^{\operatorname{arm}(u)} t^{\operatorname{leg}(u)+1}}\right) x^{\operatorname{content}(\sigma)} .
$$

\section{Hall-Littlewood polynomials of type $C_{n}$}

In this section we present a new formula for the Hall-Littlewood polynomials of type $C$ in terms of fillings of Young diagrams. This formula will be derived by compressing Schwer's formula [18] (cf. also [16]).

Let $\lambda=\left(\lambda_{1}>\ldots>\lambda_{n}>0\right)$ be a partition with $n$ distinct parts for a fixed $n \geq 2$ (this corresponds to a dominant regular weight for the root system of type $C_{n}$ ). Consider the shape $\widehat{\lambda}$ obtained from $\lambda$ by replacing each column of height $k$ with $k$ or $2 k-1$ (adjacent) copies of it, depending on the given column being the first one or not. We are representing a filling $\sigma$ of $\widehat{\lambda}$ as a concatenation of columns $C_{i j}$ and $C_{i k}^{\prime}$, where $i=1, \ldots, \lambda_{1}$, while for a given $i$ we have $j=1, \ldots, \lambda_{i}^{\prime}$ if $i>1, j=1$ if $i=1$, and $k=2, \ldots, \lambda_{i}^{\prime}$; the columns $C_{i j}$ and $C_{i k}^{\prime}$ have height $\lambda_{i}^{\prime}$. The diagram $\hat{\lambda}$ is represented in "Japanese style", like in the previous section, i.e., the heights of columns increase from left to right; more precisely, we let

$$
\sigma=\mathcal{C}^{\lambda_{1}} \ldots \mathcal{C}^{1}, \quad \text { where } \mathcal{C}^{i}:= \begin{cases}C_{i 2}^{\prime} \ldots C_{i, \lambda_{i}^{\prime}}^{\prime} C_{i 1} \ldots C_{i, \lambda_{i}^{\prime}} & \text { if } i>1 \\ C_{i 2}^{\prime} \ldots C_{i, \lambda_{i}^{\prime}}^{\prime} C_{i 1} & \text { if } i=1 .\end{cases}
$$

Note that the leftmost column is $C_{\lambda_{1}, 1}$, and the rightmost column is $C_{11}$. For an example, we refer to Section 6

Essentially, the above description says that the column to the right of $C_{i j}$ is $C_{i, j+1}$, whereas the column to the right of $C_{i k}^{\prime}$ is $C_{i, k+1}^{\prime}$. Here we are assuming that the mentioned columns exist, up to the following conventions:

$$
C_{i, \lambda_{i}^{\prime}+1}=\left\{\begin{array}{ll}
C_{i-1,2}^{\prime} & \text { if } i>1 \text { and } \lambda_{i-1}^{\prime}>1 \\
C_{i-1,1} & \text { if } i>1 \text { and } \lambda_{i-1}^{\prime}=1,
\end{array} \quad C_{i, \lambda_{i}^{\prime}+1}^{\prime}=C_{i 1} .\right.
$$


We consider the alphabet $[\bar{n}]:=\{1<\ldots<n<\bar{n}<\overline{n-1}<\ldots<\overline{1}\}$, where the barred entries are viewed as negatives, so that $-\bar{\imath}=i$. Next, we consider the set $\mathcal{T}(\widehat{\lambda}, \bar{n})$ of fillings of $\widehat{\lambda}$ with entries in $[\bar{n}]$ which satisfy the following conditions:

1. the rows are weakly decreasing from left to right;

2. no column contains two entries $a, b$ with $a= \pm b$;

3. any two adjacent columns are related as indicated below (essentially, they differ by a "signed cycle").

In order to explain the mentioned relation between adjacent columns, we consider right actions of type $C$ reflections on columns (see Section 6). For instance, $C(a, \bar{b})$ is the column obtained from $C$ by transposing the entries in positions $a, b$ and by changing their signs. Let us first explain the passage from some column $C_{i j}$ to $C_{i, j+1}$. There exist positions $1 \leq r_{1}<\ldots<r_{p}<j$ (possibly $p=0$ ) such that $C_{i, j+1}$ differs from $D=C_{i j}\left(r_{1}, \bar{\jmath}\right) \ldots\left(r_{p}, \bar{\jmath}\right)$ only in position $j$, while $C_{i, j+1}(j) \notin\left\{ \pm D(r): r \in\left[\lambda_{i}^{\prime}\right] \backslash\{j\}\right\}$ and $C_{i, j+1}(j) \leq D(j)$. To include the case $j=\lambda_{i}^{\prime}$ in this description, just replace $C_{i, j+1}$ everywhere by $C_{i, j+1}\left[1, \lambda_{i}^{\prime}\right]$ and use the conventions (2). Let us now explain the passage from some column $C_{i k}^{\prime}$ to $C_{i, k+1}^{\prime}$. There exist positions $1 \leq r_{1}<\ldots<r_{p}<k$ (possibly $p=0$ ) such that $C_{i, k+1}^{\prime}=C_{i k}^{\prime}\left(r_{1}, \bar{k}\right) \ldots\left(r_{p}, \bar{k}\right)$. This description includes the case $k=\lambda_{i}^{\prime}$, based on the conventions 22.

Let us now define the content of a filling. For this purpose, we first associate with a filling $\sigma$ a compressed version of it, namely the filling $\bar{\sigma}$ of the partition $2 \lambda$. This is defined as follows:

$$
\bar{\sigma}=\overline{\mathcal{C}}^{\lambda_{1}} \ldots \overline{\mathcal{C}}^{1}, \quad \text { where } \overline{\mathcal{C}}^{i}:=C_{i 2}^{\prime} C_{i 1},
$$

where the conventions (2) are used again. Now define content $(\sigma)=\left(m_{1}, \ldots, m_{n}\right)$, where $m_{i}$ is half the difference between the number of occurences of the entries $i$ and $\bar{\imath}$ in $\bar{\sigma}$.

We now define two statistics on fillings that will be used in our compressed formula for Hall-Littlewood polynomials. Intervals refer to the discrete set $[\bar{n}]$. Let

$$
\sigma_{a b}:= \begin{cases}1 & \text { if } a, b \geq \bar{n} \\ 0 & \text { otherwise }\end{cases}
$$

Given a sequence of integers $w$, we write $w[i, j]$ for the subsequence $w(i) w(i+1) \ldots w(j)$. We use the notation $N_{a b}(w)$ for the number of entries $w(i)$ in the interval $(a, b)$.

Given two columns $D, C$ of the same height $d$ such that $D \geq C$ in the componentwise order, we will define two statistics $N(D, C)$ and $\operatorname{des}(D, C)$ in some special cases, as specified below.

Case 0. If $D=C$, then $N(D, C):=0$ and $\operatorname{des}(D, C):=0$.

Case 1. Assume that $C=D(r, \bar{\jmath})$ with $r<j$. Let $a:=D(r)$ and $b:=D(j)$. In this case, we set

$$
N(D, C):=N_{\bar{b} a}(D[r+1, j-1])+|(\bar{b}, a) \backslash\{ \pm D(i): i=1, \ldots, j\}|+\sigma_{a b}, \quad \operatorname{des}(D, C):=1 .
$$

Case 2. Assume that $C=D\left(r_{1}, \bar{\jmath}\right) \ldots\left(r_{p}, \bar{\jmath}\right)$ where $1 \leq r_{1}<\ldots<r_{p}<j$. Let $D_{i}:=D\left(r_{1}, \bar{\jmath}\right) \ldots\left(r_{i}, \bar{\jmath}\right)$ for $i=0, \ldots, p$, so that $D_{0}=D$ and $D_{p}=C$. We define

$$
N(D, C):=\sum_{i=1}^{p} N\left(D_{i-1}, D_{i}\right), \quad \operatorname{des}(D, C):=p .
$$

Case 3. Assume that $C$ differs from $D^{\prime}:=D\left(r_{1}, \bar{\jmath}\right) \ldots\left(r_{p}, \bar{\jmath}\right)$ with $1 \leq r_{1}<\ldots<r_{p}<j$ (possibly $p=0$ ) only in position $j$, while $C(j) \notin\left\{ \pm D^{\prime}(r): r \in[d] \backslash\{j\}\right\}$ and $C(j) \leq D^{\prime}(j)$. We define

$$
N(D, C):=N\left(D, D^{\prime}\right)+N_{C(j), D^{\prime}(j)}(D[j+1, d]), \quad \operatorname{des}(D, C):=p+1 .
$$


If the height of $C$ is larger than the height $d$ of $D$ (necessarily by 1), and $N(D, C[1, d])$ can be computed as above, we let $N(D, C):=N(D, C[1, d])$ and $\operatorname{des}(D, C):=\operatorname{des}(D, C[1, d])$. Given a filling $\sigma$ in $\mathcal{T}(\widehat{\lambda}, \bar{n})$ with columns $C_{m}, \ldots, C_{1}$, we set

$$
N(\sigma):=\sum_{i=1}^{m-1} N\left(C_{i+1}, C_{i}\right)+\operatorname{inv}\left(C_{1}\right) ;
$$

here $\operatorname{inv}\left(C_{1}\right)$ denotes the number of (ordinary) inversions in $C_{1}$, that is, the number of pairs $i<j$ of positions in $C_{1}$ with $C_{1}(i)>C_{1}(j)$. Furthermore, in the mentioned case, we also set

$$
\operatorname{des}(\sigma):=\sum_{i=1}^{m-1} \operatorname{des}\left(C_{i+1}, C_{i}\right) .
$$

We can now state our new formula for the Hall-Littlewood polynomials of type $C$. We refer to Remarks 6.6 for more comments on this formula.

Theorem 3.1 Given a partition $\lambda$ with $n$ distinct parts, the Hall-Littlewood polynomial $P_{\lambda}(X ; t)$ is given by

$$
P_{\lambda}(X ; t)=\sum_{\sigma \in \mathcal{T}(\widehat{\lambda}, \bar{n})} t^{N(\sigma)}(1-t)^{\operatorname{des}(\sigma)} x^{\operatorname{content}(\sigma)} .
$$

\section{Alcove walks and Macdonald polynomials}

\subsection{Root systems}

We recall some background information on finite root systems and affine Weyl groups. Let $\mathfrak{g}$ be a complex semisimple Lie algebra, and $\mathfrak{h}$ a Cartan subalgebra, whose rank is $r$. Let $\Phi \subset \mathfrak{h}^{*}$ be the corresponding irreducible root system, $\mathfrak{h}_{\mathbb{R}}^{*} \subset \mathfrak{h}^{*}$ the real span of the roots, and $\Phi^{+} \subset \Phi$ the set of positive roots. Let $\rho:=\frac{1}{2}\left(\sum_{\alpha \in \Phi^{+}} \alpha\right)$. Let $\alpha_{1}, \ldots, \alpha_{r} \in \Phi^{+}$be the corresponding simple roots. We denote by $\langle\cdot, \cdot\rangle$ the non-degenerate scalar product on $\mathfrak{h}_{\mathbb{R}}^{*}$ induced by the Killing form. Given a root $\alpha$, we consider the corresponding coroot $\alpha^{\vee}:=2 \alpha /\langle\alpha, \alpha\rangle$ and reflection $s_{\alpha}$.

Let $W$ be the corresponding Weyl group, whose Coxeter generators are denoted, as usual, by $s_{i}:=s_{\alpha_{i}}$. The length function on $W$ is denoted by $\ell(\cdot)$. The Bruhat order on $W$ is given by its covers $w \lessdot w s_{\beta}$, where $\beta \in \Phi^{+}$, and $\ell\left(w s_{\beta}\right)=\ell(w)+1$.

The weight lattice $\Lambda$ is given by $\Lambda:=\left\{\lambda \in \mathfrak{h}_{\mathbb{R}}^{*}:\left\langle\lambda, \alpha^{\vee}\right\rangle \in \mathbb{Z}\right\}$ for any $\alpha \in \Phi$. The weight lattice $\Lambda$ is generated by the fundamental weights $\omega_{1}, \ldots, \omega_{r}$, which form the dual basis to the basis of simple coroots, i.e., $\left\langle\omega_{i}, \alpha_{j}^{\vee}\right\rangle=\delta_{i j}$. The set $\Lambda^{+}$of dominant weights is given by $\Lambda^{+}:=\left\{\lambda \in \Lambda:\left\langle\lambda, \alpha^{\vee}\right\rangle \geq 0\right\}$ for any $\alpha \in \Phi^{+}$. Let $\mathbb{Z}[\Lambda]$ be the group algebra of the weight lattice $\Lambda$, which has a $\mathbb{Z}$-basis of formal exponents $\left\{x^{\lambda}: \lambda \in \Lambda\right\}$ with multiplication $x^{\lambda} \cdot x^{\mu}:=x^{\lambda+\mu}$.

Given $\alpha \in \Phi$ and $k \in \mathbb{Z}$, we denote by $s_{\alpha, k}$ the reflection in the affine hyperplane

$$
H_{\alpha, k}:=\left\{\lambda \in \mathfrak{h}_{\mathbb{R}}^{*}:\left\langle\lambda, \alpha^{\vee}\right\rangle=k\right\} .
$$

These reflections generate the affine Weyl group $W_{\text {aff }}$ for the dual root system $\Phi^{\vee}:=\left\{\alpha^{\vee}: \quad \alpha \in \Phi\right\}$. The hyperplanes $H_{\alpha, k}$ divide the real vector space $\mathfrak{h}_{\mathbb{R}}^{*}$ into open regions, called alcoves. The fundamental alcove $A^{\circ}$ is given by

$$
A^{\circ}:=\left\{\lambda \in \mathfrak{h}_{\mathbb{R}}^{*}: 0<\left\langle\lambda, \alpha^{\vee}\right\rangle<1 \text { for all } \alpha \in \Phi^{+}\right\} .
$$

\subsection{Alcove walks}

We say that two alcoves $A$ and $B$ are adjacent if they are distinct and have a common wall. Given a pair of adjacent alcoves $A \neq B$ (i.e., having a common wall), we write $A \stackrel{\beta}{\longrightarrow} B$ if the common wall is of the form $H_{\beta, k}$ and the root $\beta \in \Phi$ points in the direction from $A$ to $B$. 
Definition 4.1 [10] An alcove path is a sequence of alcoves such that any two consecutive ones are adjacent. We say that an alcove path $\left(A_{0}, A_{1}, \ldots, A_{m}\right)$ is reduced if $m$ is the minimal length of all alcove paths from $A_{0}$ to $A_{m}$.

We need the following generalization of alcove paths.

Definition 4.2 An alcove walk is a sequence $\Omega=\left(A_{0}, F_{1}, A_{1}, F_{2}, \ldots, F_{m}, A_{m}, F_{\infty}\right)$ such that $A_{0}, \ldots, A_{m}$ are alcoves; $F_{i}$ is a codimension one common face of the alcoves $A_{i-1}$ and $A_{i}$, for $i=1, \ldots, m$; and $F_{\infty}$ is a vertex of the last alcove $A_{m}$. The weight $F_{\infty}$ is called the weight of the alcove walk, and is denoted by $\mu(\Omega)$.

The folding operator $\phi_{i}$ is the operator which acts on an alcove walk by leaving its initial segment from $A_{0}$ to $A_{i-1}$ intact and by reflecting the remaining tail in the affine hyperplane containing the face $F_{i}$. In other words, we define

$$
\phi_{i}(\Omega):=\left(A_{0}, F_{1}, A_{1}, \ldots, A_{i-1}, F_{i}^{\prime}=F_{i}, A_{i}^{\prime}, F_{i+1}^{\prime}, A_{i+1}^{\prime}, \ldots, A_{m}^{\prime}, F_{\infty}^{\prime}\right),
$$

where $A_{j}^{\prime}:=\rho_{i}\left(A_{j}\right)$ for $j \in\{i, \ldots, m\}, F_{j}^{\prime}:=\rho_{i}\left(F_{j}\right)$ for $j \in\{i, \ldots, m\} \cup\{\infty\}$, and $\rho_{i}$ is the affine reflection in the hyperplane containing $F_{i}$. Note that any two folding operators commute. An index $j$ such that $A_{j-1}=A_{j}$ is called a folding position of $\Omega$. Let $\operatorname{fp}(\Omega):=\left\{j_{1}<\ldots<j_{s}\right\}$ be the set of folding positions of $\Omega$. If this set is empty, $\Omega$ is called unfolded. Given this data, we define the operator "unfold", producing an unfolded alcove walk, by

$$
\operatorname{unfold}(\Omega)=\phi_{j_{1}} \ldots \phi_{j_{s}}(\Omega) .
$$

Definition 4.3 A folding position $j$ of the alcove walk $\Omega=\left(A_{0}, F_{1}, A_{1}, F_{2}, \ldots, F_{m}, A_{m}, F_{\infty}\right)$ is called a positive folding if the alcove $A_{j-1}=A_{j}$ lies on the positive side of the affine hyperplane containing the face $F_{j}$. Otherwise, the folding position is called a negative folding.

Let $\tau_{\lambda} \in W_{\text {aff }}$ denote the translation by $\lambda$. Recall the bijection $A \mapsto v_{A}$ between alcoves and affine Weyl group elements given by $v_{A}\left(A^{\circ}\right)=A$. We now fix a dominant weight $\lambda$ and a reduced alcove path $\Pi:=$ $\left(A_{0}, A_{1}, \ldots, A_{m}\right)$ from $A^{\circ}=A_{0}$ to the alcove $A_{m}$ corresponding to the minimal element in the coset $\tau_{\lambda} W$ under the mentioned bijection. Assume that we have

$$
A_{0} \stackrel{\beta_{1}}{\longrightarrow} A_{1} \stackrel{\beta_{2}}{\longrightarrow} \ldots \stackrel{\beta_{m}}{\longrightarrow} A_{m}
$$

where $\Gamma:=\left(\beta_{1}, \ldots, \beta_{m}\right)$ is a sequence of positive roots. This sequence, which determines the alcove path, is called a $\lambda$-chain (of roots).

We also let $r_{i}:=s_{\beta_{i}}$, and let $\widehat{r}_{i}$ be the affine reflection in the common wall of $A_{i-1}$ and $A_{i}$, for $i=1, \ldots, m$; in other words, $\widehat{r}_{i}:=s_{\beta_{i}, l_{i}}$, where $l_{i}:=\left|\left\{j \leq i: \beta_{j}=\beta_{i}\right\}\right|$ is the cardinality of the corresponding set. Given $J=\left\{j_{1}<\ldots<j_{s}\right\} \subseteq[m]:=\{1, \ldots, m\}$, we define the Weyl group element $\phi(J)$ and the weight $\mu(J)$ by

$$
\phi(J):=r_{j_{1}} \ldots r_{j_{s}}, \quad \mu(J):=\widehat{r}_{j_{1}} \ldots \widehat{r}_{j_{s}}(\lambda) .
$$

\subsection{The Ram-Yip formula for Macdonald polynomials}

Given $w \in W$ and the alcove path $\Pi$ considered above, we define the alcove path

$$
w(\Pi):=\left(w\left(A_{0}\right), w\left(A_{1}\right), \ldots, w\left(A_{m}\right)\right) .
$$

Consider the set of alcove paths $\mathcal{P}(\Gamma):=\{w(\Pi): w \in W\}$. We identify any $w(\Pi)$ with the obvious unfolded alcove walk of weight $\mu(w(\Pi)):=w(\lambda)$. Let us now consider the set of alcove walks

$$
\mathcal{F}(\Gamma):=\{\text { alcove walks } \Omega: \operatorname{unfold}(\Omega) \in \mathcal{P}(\Gamma)\} .
$$

We can encode an alcove walk $\Omega$ in $\mathcal{F}(\Gamma)$ by the pair $(w, J)$ in $W \times 2^{[m]}$, where

$$
\operatorname{fp}(\Omega)=J \quad \text { and } \quad \text { unfold }(\Omega)=w(\Pi) .
$$

Clearly, we can recover $\Omega$ from $(w, J)$ with $J=\left\{j_{1}<\ldots<j_{s}\right\}$ by $\Omega=\phi_{j_{1}} \ldots \phi_{j_{s}}(w(\Pi))$. We call a pair $(w, J)$ a folding pair, and, for simplicity, we denote the set $W \times 2^{[m]}$ of such pairs by $\mathcal{F}(\Gamma)$ as well. Given a folding pair $(w, J)$, the corresponding positive and negative foldings (viewed as a partition of $J$ ) are denoted by $J^{+}$and $J^{-}$. 
Proposition 4.4 (1) Consider a folding pair $(w, J)$ with $J=\left\{j_{1}<\ldots<j_{s}\right\}$. We have $j_{i} \in J^{+}$if and only if $w r_{j_{1}} \ldots r_{j_{i-1}}>w r_{j_{1}} \ldots r_{j_{i-1}} r_{j_{i}}$. (2) If $\Omega \mapsto(w, J)$, then $\mu(\Omega)=w(\mu(J))$.

We call the sequence $w, w r_{j_{1}}, \ldots, w r_{j_{1}} \ldots r_{j_{s}}=w \phi(J)$ the Bruhat chain associated to $(w, J)$.

We now restate the Ram-Yip formula [17] for the Macdonald polynomials $P_{\lambda}(X ; q, t)$ in terms of folding pairs. From now on we assume that the weight $\lambda$ is regular (and dominant), i.e., $\left\langle\lambda, \alpha^{\vee}\right\rangle>0$ for all positive roots $\alpha$.

Theorem 4.5 [17] Given a dominant regular weight $\lambda$, we have (based on the notation in Section 4.2]

$$
\begin{aligned}
& P_{\lambda}(X ; q, t)= \\
= & \sum_{(w, J) \in \mathcal{F}(\Gamma)} t^{\frac{1}{2}(\ell(w)-\ell(w \phi(J))-|J|)}(1-t)^{|J|}\left(\prod_{j \in J^{+}} \frac{1}{1-q^{l_{j}} t^{\left\langle\rho, \beta_{j}^{\vee}\right\rangle}}\right)\left(\prod_{j \in J^{-}} \frac{q^{l_{j}} t^{\left\langle\rho, \beta_{j}^{\vee}\right\rangle}}{1-q^{l_{j}} t^{\left\langle\rho, \beta_{j}^{\vee}\right\rangle}}\right) x^{w(\mu(J))} .
\end{aligned}
$$

\subsection{Schwer's formula for Hall-Littlewood polynomials}

Let us now consider a reduced alcove path from $A^{\circ}$ to $A^{\circ}+\lambda$. The associated chain of roots $\Gamma$, defined as in (6), will be called an extended $\lambda$-chain. All the previous definitions can be adapted to this setup. Let $\mathcal{F}_{+}(\Gamma)$ consist of the folding pairs $(w, J)$ with $J_{-}=\emptyset$, which will be called positive folding pairs.

Theorem 4.6 [16, 18] Given a dominant regular weight $\lambda$, the Hall-Littlewood polynomial $P_{\lambda}(X ; t)$ is given by

$$
P_{\lambda}(X ; t)=\sum_{(w, J) \in \mathcal{F}_{+}(\Gamma)} t^{\frac{1}{2}(\ell(w)+\ell(w \phi(J))-|J|)}(1-t)^{|J|} x^{w(\mu(J))} .
$$

\section{Compressing the Ram-Yip formula in type $A_{n-1}$}

We now restrict ourselves to the root system of type $A_{n-1}$, fow which the Weyl group $W$ is the symmetric group $S_{n}$. Permutations $w \in S_{n}$ are written in one-line notation $w=w(1) \ldots w(n)$. We can identify the space $\mathfrak{h}_{\mathbb{R}}^{*}$ with the quotient space $V:=\mathbb{R}^{n} / \mathbb{R}(1, \ldots, 1)$, where $\mathbb{R}(1, \ldots, 1)$ denotes the subspace in $\mathbb{R}^{n}$ spanned by the vector $(1, \ldots, 1)$. The action of the symmetric group $S_{n}$ on $V$ is obtained from the (left) $S_{n}$-action on $\mathbb{R}^{n}$ by permutation of coordinates. Let $\varepsilon_{1}, \ldots, \varepsilon_{n} \in V$ be the images of the coordinate vectors in $\mathbb{R}^{n}$. The root system $\Phi$ can be represented as $\Phi=\left\{\alpha_{i j}:=\varepsilon_{i}-\varepsilon_{j}: i \neq j, 1 \leq i, j \leq n\right\}$. The simple roots are $\alpha_{i}=\alpha_{i, i+1}$, for $i=1, \ldots, n-1$. The fundamental weights are $\omega_{i}=\varepsilon_{1}+\ldots+\varepsilon_{i}$, for $i=1, \ldots, n-1$. The weight lattice is $\Lambda=\mathbb{Z}^{n} / \mathbb{Z}(1, \ldots, 1)$. A dominant weight $\lambda=\lambda_{1} \varepsilon_{1}+\ldots+\lambda_{n-1} \varepsilon_{n-1}$ is identified with the partition $\left(\lambda_{1} \geq \lambda_{2} \geq \ldots \geq \lambda_{n-1} \geq \lambda_{n}=0\right)$ of length at most $n-1$. We fix such a partition $\lambda$ for the remainder of this section.

For simplicity, we use the same notation $(i, j)$ with $i<j$ for the root $\alpha_{i j}$ and the reflection $s_{\alpha_{i j}}$, which is the transposition of $i$ and $j$. Consider the following chain of roots, denoted by $\Gamma(k)$ :

$$
\begin{array}{llll}
(k, n), & (k, n-1), & \ldots, & (k, k+1), \\
(k-1, n), & (k-1, n-1), & \ldots, & (k-1, k+1), \\
& & \ldots & \\
(1, n), & (1, n-1), & \ldots, & (1, k+1)) .
\end{array}
$$

Denote by $\Gamma^{\prime}(k)$ the chain of roots obtained by removing the root $(i, k+1)$ at the end of each row. Now define a chain $\Gamma$ as a concatenation $\Gamma:=\Gamma_{\lambda_{1}} \ldots \Gamma_{2}$, where

$$
\Gamma_{j}:= \begin{cases}\Gamma^{\prime}\left(\lambda_{j}^{\prime}\right) & \text { if } j=\min \left\{i: \lambda_{i}^{\prime}=\lambda_{j}^{\prime}\right\} \\ \Gamma\left(\lambda_{j}^{\prime}\right) & \text { otherwise. }\end{cases}
$$

It is not hard to verify that $\Gamma$ is a $\lambda$-chain in the sense discussed in Section 4.2 The $\lambda$-chain $\Gamma$ is fixed for the remainder of this section. Thus, we can replace the notation $\mathcal{F}(\Gamma)$ with $\mathcal{F}(\lambda)$. 
Example 5.1 Consider $n=4$ and $\lambda=(4,3,1,0)$, for which we have the following $\lambda$-chain (the underlined pairs are only relevant in Example 5.2 below):

$$
\Gamma=\Gamma_{4} \Gamma_{3} \Gamma_{2}=(\underline{(1,4)},(1,3)|(2,4), \underline{(2,3)},(1,4), \underline{(1,3)}| \underline{(2,4)},(1,4)) .
$$

Given the $\lambda$-chain $\Gamma$ above, in Section 4.2 we considered subsets $J=\left\{j_{1}<\ldots<j_{s}\right\}$ of $[m]$, where $m$ is the length of the $\lambda$-chain. Instead of $J$, it is now convenient to use the subsequence of $\Gamma$ indexed by the positions in $J$. This is viewed as a concatenation with distinguished factors $T=T_{\lambda_{1}} \ldots T_{2}$ induced by the factorization of $\Gamma$ as $\Gamma_{\lambda_{1}} \ldots \Gamma_{2}$. The partition $J=J^{+} \sqcup J^{-}$induces partitions $T=T^{+} \sqcup T^{-}$and $T_{j}=T_{j}^{+} \sqcup T_{j}^{-}$. All the notions defined in terms of $J$ are now redefined in terms of $T$. As such, from now on we will write $\phi(T), \mu(T)$, and $|T|$, the latter being the size of $T$. If $(w, J)$ is a folding pair, we will use the same name for the corresponding pair $(w, T)$. We will use the notation $\mathcal{F}(\Gamma)$ and $\mathcal{F}(\lambda)$ accordingly. We denote by $w T_{\lambda_{1}} \ldots T_{j}$ the permutation obtained from $w$ via right multiplication by the transpositions in $T_{\lambda_{1}}, \ldots, T_{j}$, considered from left to right. This agrees with the above convention of using pairs to denote both roots and the corresponding reflections. As such, $\phi(J)$ in (7) can now be written simply $T$.

Example 5.2 We continue Example 5.1, by picking the folding pair $(w, J)$ with $w=2341 \in S_{4}$ and $J=$ $\{1,4,6,7\}$ (see the underlined positions in (11). Thus, we have

$$
T=T_{4} T_{3} T_{2}=((1,4)|(2,3),(1,3)|(2,4)) .
$$

Note that $J^{+}=\{1,7\}$ and $J^{-}=\{4,6\}$. Indeed, we have the following Bruhat chain associated to $(w, T)$, where the transposed entries are shown in bold (we represent permutations as broken columns):

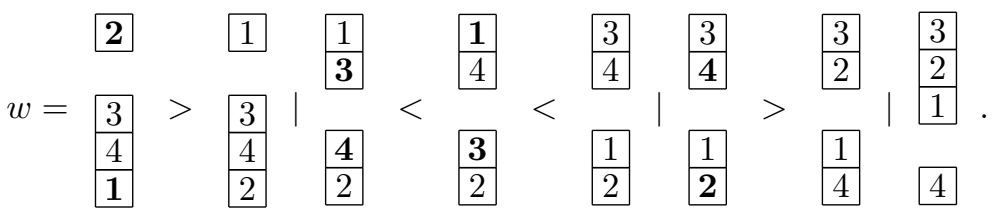

Given a folding pair $(w, T)$, we consider the permutations

$$
\pi_{j}=\pi_{j}(w, T):=w T_{\lambda_{1}} T_{\lambda_{1}-1} \ldots T_{j+1},
$$

for $j=1, \ldots, \lambda_{1}$. In particular, $\pi_{\lambda_{1}}=w$.

Definition 5.3 The filling map is the map $f$ from folding pairs $(w, T)$ to fillings $\sigma=f(w, T)$ of the shape $\lambda$, defined by $\sigma(i, j):=\pi_{j}(i)$.

Example 5.4 Given $(w, T)$ as in Example 5.2, we have

$$
f(w, T)=\begin{array}{|l|l|l|l|}
2 & 1 & 3 & 3 \\
\hline 3 & 4 & 2 \\
\hline
\end{array} \quad 1 .
$$

From now on, we assume that the partition $\lambda$ corresponds to a regular weight, i.e., $\left(\lambda_{1}>\ldots>\lambda_{n-1}>0\right)$. We will now describe the way in which the formula (1) of Haglund-Haiman-Loehr type can be obtained by compressing the Ram-Yip formula (8). Thus, Theorem 2.6 becomes a corollary of Theorem 5.5 below. We start by rewriting the Ram-Yip formula 8 in the type $A$ setup, as follows:

$$
\begin{gathered}
P_{\lambda}(X ; q, t)=\sum_{(w, T) \in \mathcal{F}(\Gamma)} t^{\frac{1}{2}(\ell(w)-\ell(w T)-|T|)}(1-t)^{|T|}\left(\prod_{j,(i, k) \in T_{j}^{+}} \frac{1}{1-q^{\operatorname{arm}(-j+1,-i)} t^{k-i}}\right) \times \\
\times\left(\prod_{j,(i, k) \in T_{j}^{-}} \frac{q^{\operatorname{arm}(-j+1,-i)} t^{k-i}}{1-q^{\operatorname{arm}(-j+1,-i)} t^{k-i}}\right) x^{w(\mu(T))} .
\end{gathered}
$$


Theorem 5.5 We have $f(\mathcal{F}(\lambda))=\mathcal{T}(\lambda, n)$. Given any $\sigma \in \mathcal{T}(\lambda, n)$ and any $(w, T) \in f^{-1}(\sigma)$, we have $\operatorname{content}(f(w, T))=w(\mu(T))$. Furthermore, the following compression formula holds for any $\sigma \in \mathcal{T}(\lambda, n)$ :

$$
\begin{gathered}
\sum_{(w, T) \in f^{-1}(\sigma)} t^{\frac{1}{2}(\ell(w)-\ell(w T)-|T|)}(1-t)^{|T|}\left(\prod_{j,(i, k) \in T_{j}^{+}} \frac{1}{1-q^{\operatorname{arm}(i, j-1)} t^{k-i}}\right) \times \\
\times\left(\prod_{j,(i, k) \in T_{j}^{-}} \frac{q^{\operatorname{arm}(i, j-1)} t^{k-i}}{1-q^{\operatorname{arm}(i, j-1)} t^{k-i}}\right)=t^{n(\lambda)-\operatorname{inv}(\sigma)} q^{\operatorname{maj}(\sigma)}\left(\prod_{u \in \operatorname{Diff}(\sigma)} \frac{1-t}{1-q^{\operatorname{arm}(u)} t^{\operatorname{leg}(u)+1}}\right) .
\end{gathered}
$$

\section{Compressing Schwer's formula in type $C_{n}$}

We now restrict ourselves to the root system of type $C_{n}$. We can identify the space $\mathfrak{h}_{\mathbb{R}}^{*}$ with $V:=\mathbb{R}^{n}$, the coordinate vectors being $\varepsilon_{1}, \ldots, \varepsilon_{n}$. The root system $\Phi$ can be represented as $\Phi=\left\{ \pm \varepsilon_{i} \pm \varepsilon_{j}: 1 \leq i<j \leq n\right\} \cup\left\{ \pm 2 \varepsilon_{i}\right.$ : $1 \leq i \leq n\}$. The simple roots are $\alpha_{i}=\varepsilon_{i}-\varepsilon_{i+1}$, for $i=1, \ldots, n-1$ and $\alpha_{n}=2 \varepsilon_{n}$. The fundamental weights are $\omega_{i}=\varepsilon_{1}+\ldots+\varepsilon_{i}$, for $i=1, \ldots, n$. The weight lattice is $\Lambda=\mathbb{Z}^{n}$. A dominant weight $\lambda=\lambda_{1} \varepsilon_{1}+\ldots+\lambda_{n} \varepsilon_{n}$ is identified with the partition $\left(\lambda_{1} \geq \lambda_{2} \geq \ldots \geq \lambda_{n} \geq 0\right)$ of length at most $n$. We fix such a partition $\lambda$ for the remainder of this section.

The corresponding Weyl group $W$ is the group of signed permutations $B_{n}$. Such permutations are bijections $w$ from $[\bar{n}]:=\{1<\ldots<n<\bar{n}<\overline{n-1}<\ldots<\overline{1}\}$ to $[\bar{n}]$ satisfying $w(\bar{\imath})=\overline{w(i)}$. We use the window notation $w=w(1) \ldots w(n)$. The group $B_{n}$ acts on $V$ as usual, by permuting the coordinate vectors and by changing their signs.

For simplicity, we use the same notation $(i, j)$ with $1 \leq i<j \leq n$ for the positive root $\varepsilon_{i}-\varepsilon_{j}$ and the corresponding reflection, which, in the window notation, is the transposition of entries in positions $i$ and $j$. Similarly, we denote by $(i, \bar{\jmath})$, again for $i<j$, the positive root $\varepsilon_{i}+\varepsilon_{j}$ and the corresponding reflection; in the window notation, the latter is the transposition of entries in positions $i$ and $j$ followed by the sign change of those entries. Finally, we denote by $(i, \bar{l})$ the positive root $2 \varepsilon_{i}$ and the corresponding reflection, which is the sign change in position $i$.

Let

$$
\Gamma(k):=\Gamma_{2}^{\prime} \ldots \Gamma_{k}^{\prime} \Gamma_{1}(k) \ldots \Gamma_{k}(k)
$$

where

$$
\begin{aligned}
& \Gamma_{j}^{\prime}:=((1, \bar{\jmath}),(2, \bar{\jmath}), \ldots,(j-1, \bar{\jmath})),
\end{aligned}
$$

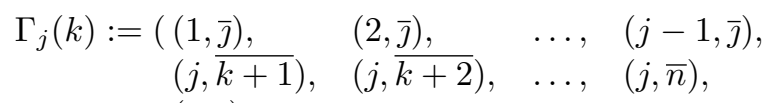

$$
\begin{aligned}
& (j, \bar{\jmath}) \text {, } \\
& (j, n), \quad(j, n-1), \quad \ldots, \quad(j, k+1)) .
\end{aligned}
$$

It is not hard to see that $\Gamma(k)$ is an extended $\omega_{k}$-chain, in the sense discussed in Section 4.4 Hence, we can construct an extended $\lambda$-chain as a concatenation $\Gamma:=\Gamma^{\lambda_{1}} \ldots \Gamma^{1}$, where

$$
\Gamma^{i}=\Gamma\left(\lambda_{i}^{\prime}\right)=\Gamma_{i 2}^{\prime} \ldots \Gamma_{i, \lambda_{i}^{\prime}}^{\prime} \Gamma_{i 1} \ldots \Gamma_{i, \lambda_{i}^{\prime}}, \quad \text { and } \Gamma_{i j}=\Gamma_{j}\left(\lambda_{i}^{\prime}\right), \quad \Gamma_{i j}^{\prime}=\Gamma_{j}^{\prime} .
$$

This extended $\lambda$-chain is fixed for the remainder of this section. Thus, we can replace the notation $\mathcal{F}_{+}(\Gamma)$ with $\mathcal{F}_{+}(\lambda)$.

Example 6.1 Consider $n=3$ and $\lambda=(3,2,1)$, for which we have the extended $\lambda$-chain below. The factorization of $\Gamma$ into subchains is indicated by vertical bars, while the double vertical bars separate the subchains corresponding to different columns. The underlined pairs are only relevant in Example 6.2 below.

$$
\Gamma=\Gamma_{31}\left\|\Gamma_{22}^{\prime} \Gamma_{21} \Gamma_{22}\right\| \Gamma_{12}^{\prime} \Gamma_{13}^{\prime} \Gamma_{11} \Gamma_{12} \Gamma_{13}=
$$




$$
\begin{aligned}
= & ((1, \overline{2}), \underline{(1, \overline{3})},(1, \overline{1}),(1,3),(1,2)|| \underline{(1, \overline{2})}|(1, \overline{3}),(1, \overline{1}),(1,3)|(1, \overline{2}),(2, \overline{3}), \underline{(2, \overline{2})}, \underline{(2,3)} \| \\
& (1, \overline{2})|(1, \overline{3}),(2, \overline{3})|(1, \overline{1})|(1, \overline{2}),(2, \overline{2})|(1, \overline{3}),(2, \overline{3}),(3, \overline{3})) .
\end{aligned}
$$

Given the extended $\lambda$-chain $\Gamma$ above, in Section 4.2 we considered subsets $J=\left\{j_{1}<\ldots<j_{s}\right\}$ of $[m]$, where $m$ is the length of $\Gamma$. Instead of $J$, it is now convenient to use the subsequence of $\Gamma$ indexed by the positions in $J$. This is viewed as a concatenation with distinguished factors $T_{i j}$ and $T_{i k}^{\prime}$ induced by the factorization 12 of $\Gamma$. All the notions defined in terms of $J$ are now redefined in terms of $T$. As such, from now on we will write $\phi(T), \mu(T)$, and $|T|$, the latter being the size of $T$. If $(w, J)$ is positive folding pair, we will use the same name for the corresponding pair $(w, T)$. We denote by $w T_{\lambda_{1}, 1} \ldots T_{i j}$ and $w T_{\lambda_{1}, 1} \ldots T_{i k}^{\prime}$ the permutations obtained from $w$ via right multiplication by the reflections in $T_{\lambda_{1}, 1}, \ldots, T_{i j}$ and $T_{\lambda_{1}, 1}, \ldots, T_{i k}^{\prime}$, considered from left to right. This agrees with the above convention of using pairs to denote both roots and the corresponding reflections. As such, $\phi(J)$ in 77 can now be written simply $T$.

Example 6.2 We continue Example 6.1 by picking the positive folding pair $(w, J)$ with $w=\overline{1} \overline{2} \overline{3} \in B_{3}$ and $J=\{2,6,12,13\}$ (see the underlined positions in (13)). Thus, we have

$$
T=T_{31}\left\|T_{22}^{\prime} T_{21} T_{22}\right\| T_{12}^{\prime} T_{13}^{\prime} T_{11} T_{12} T_{13}=((1, \overline{3})\|(1, \overline{2})|\quad|(2, \overline{2}),(2,3)\||||\quad| \quad .
$$

We have the following decreasing Bruhat chain associated to $(w, T)$, where the modified entries are shown in bold (we represent signed permutations as broken columns, as in Example 5.2, and we display the splitting of the chain into subchains induced by the above splitting of $T$ ):

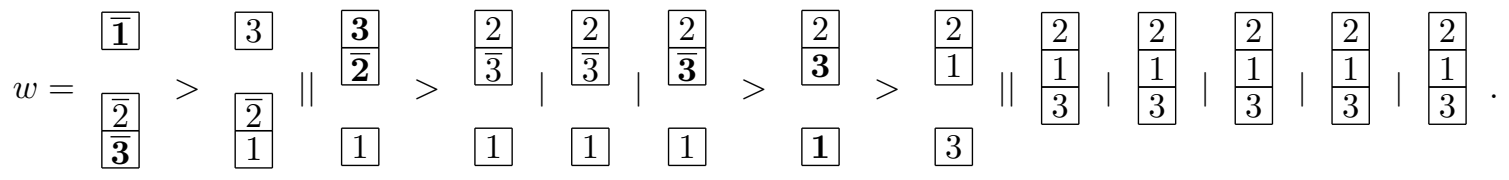

Given a positive folding pair $(w, T)$, with $T$ split into factors $T_{i j}$ and $T_{i k}^{\prime}$ as above, we consider the signed permutations

$$
\pi_{i j}=\pi_{i j}(w, T):=w T_{\lambda_{1}, 1} \ldots T_{i, j-1}, \quad \pi_{i k}^{\prime}=\pi_{i k}^{\prime}(w, T):=w T_{\lambda_{1}, 1} \ldots T_{i, k-1}^{\prime} ;
$$

when undefined, $T_{i, j-1}$ and $T_{i, k-1}^{\prime}$ are given by conventions similar to $(2)$, based on the corresponding factorization (12) of the extended $\lambda$-chain $\Gamma$. In particular, $\pi_{\lambda_{1}, 1}=w$.

Let us now recall the notation in Section 3 ,

Definition 6.3 The filling map is the map $\widehat{f}$ from positive folding pairs $(w, T)$ to fillings $\sigma=\widehat{f}(w, T)$ of the shape $\widehat{\lambda}$, defined by $C_{i j}=\pi_{i j}\left[1, \lambda_{i}^{\prime}\right]$ and $C_{i k}^{\prime}=\pi_{i k}^{\prime}\left[1, \lambda_{i}^{\prime}\right]$.

Example 6.4 Given $(w, T)$ as in Example 6.2, we have

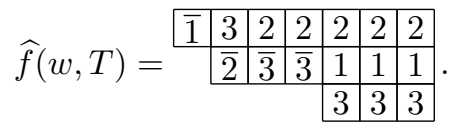

From now on, we assume that the partition $\lambda$ corresponds to a regular weight, i.e., $\left(\lambda_{1}>\ldots>\lambda_{n}>0\right)$. We will now describe the way in which the formula (4) can be obtained by compressing Schwer's formula (9). Thus, Theorem 3.1 becomes a corollary of the theorem below.

Theorem 6.5 We have $\widehat{f}\left(\mathcal{F}_{+}(\lambda)\right)=\mathcal{T}(\widehat{\lambda}, \bar{n})$. Given any $\sigma \in \mathcal{T}(\widehat{\lambda}, \bar{n})$ and $(w, T) \in \widehat{f}^{-1}(\sigma)$, we have $w(\mu(T))=$ content $(\widehat{f}(w, T))$. Furthermore, the following compression formula holds for any $\sigma \in \mathcal{T}(\widehat{\lambda}, \bar{n})$ :

$$
\sum_{(w, T) \in \widehat{f}^{-1}(\sigma)} t^{\frac{1}{2}(\ell(w)+\ell(w \phi(T))-|T|)}(1-t)^{|T|}=t^{N(\sigma)}(1-t)^{\operatorname{des}(\sigma)} .
$$


Remarks 6.6 (1) The Kashiwara-Nakashima tableaux [6] of shape $\lambda$ index the basis elements of the irreducible representation of $\mathfrak{s p}_{2 n}$ of highest weight $\lambda$. These tableaux correspond precisely to the surviving fillings in our formula (4) when we set $t=0$. More precisely, the map $\sigma \mapsto \bar{\sigma}$ (see (3) ) is a bijection between the fillings $\sigma$ in $\mathcal{T}(\widehat{\lambda}, \bar{n})$ with $N(\sigma)=0$ and the "doubled" versions of the type $C$ Kashiwara-Nakashima tableaux of shape $\lambda$.

(2) In some special cases, the statistic $N(\sigma)$ is essentially the Haglund-Haiman-Loehr "inv" statistic (extended naturally to our signed fillings), as explained below. Let $\sigma$ in $\mathcal{T}(\widehat{\lambda}, \bar{n})$ be a filling satisfying the following properties: (1) $C_{i, j+1}^{\prime}=C_{i, j}^{\prime}$ for all $i$ and $j=1, \ldots, \lambda_{i}^{\prime}$; (2) $C_{i, j+1}$ only differs from $C_{i j}$ in position $j$. Let $\widetilde{\sigma}$ be the filling of $\lambda$ given by $\widetilde{\sigma}:=C_{\lambda_{1}, 1} C_{\lambda_{1}-1,1} \ldots C_{11}$. Then $N(\sigma)=n(\lambda)-\operatorname{inv}(\widetilde{\sigma})$, where $n(\lambda):=\sum_{i}(i-1) \lambda_{i}$ and $\operatorname{inv}(\sigma)$ is the Haglund-Haiman-Loehr "inv" statistic, cf. Remark2.1 and Definition 2.5

\section{Acknowledgements}

The author was partially supported by the National Science Foundation grant DMS-0701044. The author is grateful to Jim Haglund for helpful discussions.

\section{References}

[1] S. Assaf. A combinatorial proof of LLT and Macdonald positivity, 2008. http://www-math.mit.edu/ sassaf.

[2] S. Gaussent and P. Littelmann. LS-galleries, the path model and MV-cycles. Duke Math. J., 127:35-88, 2005.

[3] I. Grojnowski and M. Haiman. Affine Hecke algebras and positivity of LLT and Macdonald polynomials, 2007. http://math.berkeley.edu/ mhaiman.

[4] J. Haglund, M. Haiman, and N. Loehr. A combinatorial formula for Macdonald polynomials. J. Amer. Math. Soc., 18:735-761, 2005.

[5] J. Haglund, M. Haiman, and N. Loehr. A combinatorial formula for nonsymmetric Macdonald polynomials. Amer. J. Math., 130:359-383, 2008.

[6] M. Kashiwara and T. Nakashima. Crystal graphs for representations of the $q$-analogue of classical Lie algebras. J. Algebra, 165:295-345, 1994.

[7] A. Lascoux. Schubert and Macdonald polynomials, a parallel, 2008. http://phalanstere.univ-mlv.fr/ al.

[8] C. Lenart. Haglund-Haiman-Loehr type formulas for Hall-Littlewood polynomials of type $B$ and $C$. arXiv:math/0904.2407.

[9] C. Lenart. On combinatorial formulas for Macdonald polynomials. Adv. Math., 220:324-340, 2009.

[10] C. Lenart and A. Postnikov. Affine Weyl groups in $K$-theory and representation theory. Int. Math. Res. Not., pages 1-65, 2007. Art. ID rnm038.

[11] C. Lenart and A. Postnikov. A combinatorial model for crystals of Kac-Moody algebras. Trans. Amer. Math. Soc., 360:4349-4381, 2008.

[12] P. Littelmann. A Littlewood-Richardson rule for symmetrizable Kac-Moody algebras. Invent. Math., 116:329-346, 1994.

[13] P. Littelmann. Paths and root operators in representation theory. Ann. of Math. (2), 142:499-525, 1995.

[14] I. Macdonald. Schur functions: theme and variations. In Séminaire Lotharingien de Combinatoire (SaintNabor, 1992), volume 498 of Publ. Inst. Rech. Math. Av., pages 5-39. Univ. Louis Pasteur, Strasbourg, 1992. 
[15] I. Macdonald. Orthogonal polynomials associated with root systems. Sém. Lothar. Combin., 45:Art. B45a, 40 pp. (electronic), 2000/01.

[16] A. Ram. Alcove walks, Hecke algebras, spherical functions, crystals and column strict tableaux. Pure Appl. Math. Q., 2:963-1013, 2006.

[17] A. Ram and M. Yip. A combinatorial formula for Macdonald polynomials. arXiv: math/0803.1146.

[18] C. Schwer. Galleries, Hall-Littlewood polynomials, and structure constants of the spherical Hecke algebra. Int. Math. Res. Not., pages Art. ID 75395, 31, 2006. 